\title{
Estimation of effective elastic moduli of random structure composites by the method of fundamental solutions
}

\author{
Valeriy A. Buryachenko* \\ Micromechanics and Composites LLC, 2520 Hingham Lane, Dayton, OH 45459, USA
}

\begin{abstract}
One considers linearly elastic composite media, which consist of a homogeneous matrix containing a statistically homogeneous random set of aligned homogeneous heterogeneities of non canonical shape. Effective elastic moduli as well as the first statistical moments of stresses in the phases are estimated trough the averaged boundary integrals over the inclusion boundaries. The modified popular micromechanical models are based on the numerical solution for one inhomogeneity inside the infinite matrix loaded by remote homogeneous effective field. This solution is obtained by a meshfree method based on fundamental solutions basis functions for a transmission problem in linear elasticity. The problem here addressed, consists in computing the displacement and traction fields of an elastic object, which has piecewise constant Lamé coefficients, from a given displacement (or stress) field on the infinity. The main properties of the method are analyzed and illustrated with several numerical simulations in $2 \mathrm{D}$ domains.
\end{abstract}

Keywords: Boundary integral equations, Method of fundamental solutions, Mechanics of deformable solids, Homogenization, determination of effective properties

\section{1. Introduction}

The prediction of the behavior of composite materials in terms of the mechanical properties of constituents and their microstructure is a central problem of micromechanics, which is evidently reduced to the estimation of stress fields 5 in the constituents. Appropriate, but by no means exhaustive, references for the estimation of effective elastic moduli of statistically homogeneous media are provided by the reviews [1-8]. It appears today that variants of the effective medium method $[9,10]$ and the mean field method $[11,12]$ are the most popular

\footnotetext{
* Corresponding author

Email address: buryach@aol.com (Valeriy A. Buryachenko)
}

Preprint submitted to Engineering Analysis with Boundary Elements LATEX TemplatesSeptember 13, 2015

(C) 2015. This manuscript version is made available under the Elsevier user license

http://www.elsevier.com/open-access/userlicense/1.0/ 
and widely used methods. Recently a new method has become known, namely the multiparticle effective field method (MEFM) was put forward and developed (see for references Buryachenko [6]). The MEFM is based on the theory of functions of random variables and Green's functions. Within this method one constructs a hierarchy of statistical moment equations for conditional averages of the stresses in the inclusions. The hierarchy is then cut by introducing the 15 notion of an effective field. This way the interaction of different inclusions is taken into account. The popular schemes of micromechanical analysis are based on numerical solutions for estimation of stress (or strain) distribution tensor for single (at least) inclusion inside infinite matrix subjected to so-called effective field.

20 Obtaining analytical solutions is not feasible in general even for a finite number of interacting particles, so various numerical methods have been developed, mostly based on the finite element analysis (FEA) and boundary integral equation (BIE) technique. A distinct advantage of the BIE compared to the FEA is that the BIEs require meshing only the boundary surface of computational 25 domain as opposed to the entire 3-D domain for FEA. In the BIE singular forces distributed, e.g., on the surface of particles, depend on the external field, thus yielding an integral equation for the singularity strengths. The most popular formulation is the direct one where the variables of interest are the surface displacement and traction fields that leads to a set of ill-conditioned linear sys-

so tems on discretization. The indirect formulation (e.g. the completed double layer boundary element method, see $[13,14]$ ) results in a set of integral equations of the second kind, and therefore is numerically well-behaved. Although this formulation is more complicated than the direct method, however, it leads to an unconditionally convergent iterative scheme.

35 Despite the FEM's and BEM's popularity, there are many problems (e.g. meshing surface in 3D, computing of singular integrals) where it is desirable to improve the efficiency of traditional methods. In the alternative (proposed by Trefftz), the trial functions must satisfy the governing equation exactly and the error in the satisfaction of the boundary conditions is minimised. As a conse40 quence, it is interesting to develop some alternative so-called meshless methods (see [15] for an overview and historical background on the subject) of the local boundary integral equation, boundary knot method, boundary collocation method, non-dimensional dynamic influence functions method, and the method of fundamental solutions (MFS) belonging to a boundary method for solving boundary value problems, which can be recognized as a discrete type of the indirect boundary element method with a concentrated source instead of distribution (see for references [16-19]). In this study, we will bring the MFS systematically for a single inclusion inside the infinite homogeneous matrix subjected to homogeneous loading. The MFS belonging to the BIE technique has a very so attractive option since it is truly meshless, simple to program, and is able to take into account a sharp changes in geometry. The MFS and related methods over the last years have found extensive application in computing solutions to a broad range of problems $[20,21]$.

In the MFS the solution is approximated by a finite linear combination 
of fundamental solutions (FS, assumed to be explicitly defined) with respect to source points which are positioned outside the solution domain. The unknown coefficients in the mentioned linear approximation can be determined by matching the boundary conditions in a variety of ways, the simplest (and most popular) being by collocation on a set of physical boundary points. In contrast

60 to domain discretization methods such as the finite element (FEM), the MFS is a boundary method which means that only the boundary of the solution domain needs to be considered. Moreover, the MFS is a boundary-type meshless method which does not need a boundary element mesh, either for purposes of interpolation of the trial and test functions of the solution variables, or for the

65 integration, and only a set of source points is required for the discretization of the problem being analyzed. However, unlike the BEM, no potentially troublesome integration is required in the MFS due to the placement of source points outside the solution domain when the singular integrals are avoided. At last MFS is adaptive in the sense that it can take into account sharp changes in 70 the solution and in the geometry of the domain and can easily handle complex boundary conditions. Despite the mentioned advantages, a few disadvantages are that the positioning of the source points are preassigned and also the resulting system of algebraic equations is ill-conditioned that leads to oscillation of the convergence curve of the numerical solution when a large number of source 75 points are used. Optimization of source points allocation substantially reduces computational time while some regularization methods, such as the damped singular value decomposition, the truncated singular value decomposition or the Tikhonov [22] regularization, can be used to mitigate the ill-conditioned effect (see [23]). The next step is checking the approximation in other (not collocaso tion) points at the physical boundary (or interface) surface. The strain and stress field variables outside the boundary can be obtained directly when the derivatives are analytically calculated from the MFS expansion representation over the source points with the coefficients found by matching the boundary condition at the given collocation points at the physical surface.

85 Thus, the stress distributions inside a single inclusions inside infinite matrix are assumed to be found. This solution then can be incorporated into the one or another general framework of analytical micromechanics for self-consistent estimations of so-called effective field (see for details Buryachenko [6]). However, all mentioned methods are based on the effective field hypothesis $(\mathrm{EFH}$, even $9_{0}$ if the term "effective field hypothesis" was not indicated) according to which each inclusion is located inside a homogeneous so-called effective field (see for references [6]). Effective field hypothesis is apparently the most fundamental, most prospective, and most exploited concept of micromechanics. This concept has directed a development of micromechanics over the last sixty years and made

95 a contribution to their progress incompatible with any another concept. The idea of effective field was added by the hypothesis of "ellipsoidal symmetry" for the distribution of inclusions attributed to Willis [24]. However, Buryachenko $[25,26]$ has proved that the EFH is a central one and other concepts play a satellite role providing the conditions for application of the EFH.

${ }_{100}$ The paper is organized as follow. In Section 2 we present the basic field 
equations of linear elasticity, notations, statistical description of the composite microstructure as well as representation of the effective properties through the surface integrals over the inclusion boundaries. In Section 3, one presents the method of fundamental solution (MFS) adapted to the solution for one homogeneous noncanonical inclusion inside the infinite homogeneous matrix. The known micromechanical method of the effective field (MEF, see for references [6]) and Mori-Tanaka method (MTM, $[11,12])$ are presented in Section 4 with the stress concentration factors and effective compliances expressed though the boundary integral of statistical averages over the boundary inclusions. In Sec110 tion 5 we estimate the numerical errors of both the different versions of the MFS and the different choices of the source sets. The numerical evaluation of the effective Young modulus are shown for a statistically homogeneous composites reinforced by aligned identical homogeneous heterogeneities of noncanonical shape.

\section{Preliminaries}

Let a full space $R^{d}$ with a space dimensionality $d(d=2$ and $d=3$ for $2-D$ and $3-D$ problems, respectively) contains a homogeneous matrix $v^{(0)}$ and, in general, a statistically inhomogeneous set $X=\left(v_{i}\right)$ of heterogeneities $v_{i}$ with indicator functions $V_{i}$ and bounded by the closed smooth surfaces $\Gamma_{i}:=\partial v_{i}$

${ }_{120}(i=1,2, \ldots)$ defined by the relations $\Gamma_{i}(\mathbf{x})=0\left(\mathbf{x} \in \Gamma_{i}\right), \Gamma_{i}(\mathbf{x})>0\left(\mathbf{x} \in v_{i}\right)$, and $\Gamma_{i}(\mathbf{x})<0\left(\mathbf{x} \notin v_{i}\right)$. It is assumed that the heterogeneities can be grouped into components (phases) $v^{(q)} \quad(q=1,2, \ldots, N)$ with identical mechanical and geometrical properties (such as the shape, size, orientation, and microstructure of heterogeneities).

\subsection{Basic equations}

Let a linear elastic body occupy an open bounded domain $w \subset R^{d}$ with a smooth boundary $\Gamma$ and with an indicator function $W$ and space dimensionality $d(d=2$ and $d=3$ for $2-D$ and $3-D$ problems, respectively). The domain $w$ contains a homogeneous matrix $v^{(0)}$ and a statistically inhomogeneous set ${ }_{130} X=\left(v_{i}, V_{i}, \mathbf{x}_{i}\right)$ of inclusions $v_{i}$ with indicator functions $V_{i}$ and centers $\mathbf{x}_{i}$. It is assumed that the inclusions can be grouped into component (phase) $v^{(1)}$ with identical mechanical and geometrical properties (such as the shape, size, orientation, and microstructure of inclusions). For the sake of definiteness, in the 2- $D$ case we will consider a plane-strain problem. At first no restrictions are imposed on the elastic symmetry of the phases or on the geometry of the inclusions ${ }^{1}$.

\footnotetext{
${ }^{1}$ It is known that for $2-D$ problems the plane-strain state is only possible for material symmetry no lower than orthotropic (see e.g. [27]) that will be assumed hereafter in $2-D$ case.
} 
The problem is governed by the local equations of elastostatics of composites

$$
\begin{aligned}
\nabla \boldsymbol{\sigma}(\mathbf{x}) & =\mathbf{0} \\
\boldsymbol{\sigma}(\mathbf{x}) & =\mathbf{L}(\mathbf{x}) \varepsilon(\mathbf{x}), \text { or } \quad \varepsilon(\mathbf{x})=\mathbf{M}(\mathbf{x}) \boldsymbol{\sigma}(\mathbf{x}), \\
\varepsilon(\mathbf{x}) & =\left[\nabla \otimes \mathbf{u}+(\nabla \otimes \mathbf{u})^{\top}\right] / 2, \quad \nabla \times \varepsilon(\mathbf{x}) \times \nabla=\mathbf{0}
\end{aligned}
$$

where $(.)^{\top}$ denotes transposition, $\otimes$ and $\times$ are the tensor and vector products. $\mathbf{L}(\mathbf{x})$ and $\mathbf{M}(\mathbf{x}) \equiv \mathbf{L}(\mathbf{x})^{-1}$ are the known stiffness and compliance fourth-order tensors, and the common notation for contracted products has been employed.

In particular, for isotropic constituents the stiffness tensor $\mathbf{L}$ is given in terms of the local bulk modulus $k$ and the shear modulus $\mu$ : $\mathbf{L}=(d k, 2 \mu) \equiv$ $d k \mathbf{N}_{1}+2 \mu \mathbf{N}_{2}, \mathbf{N}_{1}=\boldsymbol{\delta} \otimes \boldsymbol{\delta} / d, \mathbf{N}_{2}=\mathbf{I}-\mathbf{N}_{1}(d=2$ or 3$) ; \boldsymbol{\delta}$ and $\mathbf{I}$ are the unit second-order and fourth-order tensors. For the fiber composites it is the planestrain bulk modulus $k_{[2]}$ - instead of the 3 -D bulk modulus $k_{[3]}$ - that plays the significant role: $k_{[2]}=k_{[3]}+\mu_{[3]} / 3, \mu_{[2]}=\mu_{[3]}$. The tensors $\mathbf{g}(\mathbf{g}=\mathbf{L}, \mathbf{M})$ of material properties are piecewise constant and decomposed as $\mathbf{g} \equiv \mathbf{g}^{(0)}+\mathbf{g}_{1}(\mathbf{x})=$ $\mathbf{g}^{(0)}+\mathbf{g}_{1}^{(1)}(\mathbf{x})$ where $\mathbf{g}^{(0)}=$ const, $\mathbf{g}(\mathbf{x}) \equiv \mathbf{g}^{(0)}$ at $\mathbf{x} \in v^{0}$ and $\mathbf{g}_{1}^{(1)}(\mathbf{x}) \equiv \mathbf{g}_{1}^{(1)}$ is a homogeneous function of the $\mathbf{x} \in v^{(1)}$ :

$$
\mathbf{L}_{1}^{(1)}(\mathbf{x})=\mathbf{L}_{1}^{(1)} \equiv \text { const. at } \mathbf{x} \in v^{(1)} .
$$

The upper index ${ }^{(m)}$ indicates the components and the lower index $i$ indicates the individual inclusions; $v^{(0)}=w \backslash v, v \equiv v^{(1)}, V(\mathbf{x})=V^{(1)}=\sum V_{i}(\mathbf{x})$, and $V^{(1)}(\mathbf{x})$ and $V_{i}(\mathbf{x})$ are the indicator functions of $v^{(1)}$ and $v_{i}$, respectively.

The introduction of jumps of material properties allows one to define the stress $\boldsymbol{\tau}$ and strain $\boldsymbol{\eta}$ polarization tensors by two equivalent ways $\left(\mathbf{x} \in R^{d}\right)$

$$
\begin{aligned}
& \boldsymbol{\tau}(\mathbf{x})=\mathbf{L}_{1}(\mathbf{x}) \boldsymbol{\varepsilon}(\mathbf{x}), \quad \boldsymbol{\eta}(\mathbf{x})=\mathbf{M}_{1}(\mathbf{x}) \boldsymbol{\sigma}(\mathbf{x}), \quad \boldsymbol{\tau}=-\mathcal{L}^{(0)} \boldsymbol{\eta}, \\
& \boldsymbol{\tau}(\mathbf{x})=\boldsymbol{\sigma}(\mathbf{x})-\mathbf{L}^{(0)} \boldsymbol{\varepsilon}(\mathbf{x}), \quad \boldsymbol{\eta}(\mathbf{x})=\boldsymbol{\varepsilon}(\mathbf{x})-\mathbf{M}^{(0)} \boldsymbol{\sigma}(\mathbf{x}),
\end{aligned}
$$

which are simply a notational convenience and vanish inside the matrix $\boldsymbol{\tau}(\mathbf{x}) \equiv$ $\boldsymbol{\eta}(\mathbf{x}) \equiv \mathbf{0}\left(\mathbf{x} \in v^{(0)}\right)$. Hereafter $\mathbf{M}_{1}(\mathbf{y})$ is the jumps of $\mathbf{M}^{(k)}$ inside the component $v^{(k)}(k=0, \ldots, N)$ with respect to the $\mathbf{M}^{(0)}$, respectively. It is interesting, the popular polarization tensors (5) (see, e.g., Willis, [1]) are attributed to Hashin ${ }_{150}$ and Shtrikman [28] and Hill [29] who introduced, in fact, these notions in the equivalent form (6). For both local and nonlocal elasticity theory, Eqs. (5) and (6) are are also equivalent; however, the form (6) is preferable for subsequent manipulation because Eq. (6) does not explicitly use the constitutive equation (2) for the heterogeneities $\mathbf{x} \in v$.

We assume that the phases are perfectly bonded, so that the displacements and the traction components are continuous across the interphase boundaries, i.e.

$$
[[\mathbf{u}]]=\mathbf{0}, \quad\left[\left[\boldsymbol{\sigma} \mathbf{n}^{\mathrm{int}}\right]\right]=\mathbf{0}
$$

on the interface boundary $\Gamma^{\text {int }}$ where $\mathbf{n}^{\text {int }}$ is the normal vector on $\Gamma^{\text {int }}$ and $[[()]$. is the jump operator. The traction $\mathbf{t}(\mathbf{x})=\boldsymbol{\sigma}(\mathbf{x}) \mathbf{n}(\mathbf{x})$ acting on any plane with 
the normal $\mathbf{n}(\mathbf{x})$ through the point $\mathbf{x}$ can be represented in terms of displacements $\mathbf{t}(\mathbf{x})=\hat{\mathbf{t}}(\mathbf{n}, \nabla) \mathbf{u}(\mathbf{x})$, where $\hat{t}_{i k}(\mathbf{n}, \nabla)=L_{i j k l} n_{j}(\mathbf{x}) \partial / \partial x_{l}$ is the conormal derivative operator. The boundary conditions at the interface boundary will be considered together with the mixed boundary conditions on $\Gamma$ with the unit outward normal $\mathbf{n}^{\Gamma}$

$$
\mathbf{u}(\mathbf{x})=\mathbf{u}^{\Gamma}(\mathbf{x}), \quad \mathbf{x} \in \Gamma_{u}, \quad \boldsymbol{\sigma}(\mathbf{x}) \mathbf{n}^{\Gamma}(\mathbf{x})=\mathbf{t}^{\Gamma}(\mathbf{x}), \quad \mathbf{x} \in \Gamma_{t},
$$

where $\Gamma_{u}$ and $\Gamma_{t}$ are prescribed displacement and traction boundaries such that $\Gamma_{u} \cup \Gamma_{t}=\Gamma, \Gamma_{u} \cap \Gamma_{t}=\emptyset . \quad \mathbf{u}^{\Gamma}(\mathbf{x})$ and $\mathbf{t}^{\Gamma}(\mathbf{x})$ are, respectively, prescribed displacement on $\Gamma_{u}$ and traction on $\Gamma_{t}$. Of special practical interest are the homogeneous boundary conditions

$$
\begin{aligned}
\mathbf{u}^{\Gamma}(\mathbf{x}) & =\varepsilon^{\Gamma} \mathbf{x}, \varepsilon^{\Gamma} \equiv \text { const. }, \mathbf{x} \in \Gamma, \\
\mathbf{t}^{\Gamma}(\mathbf{x}) & =\boldsymbol{\sigma}^{\Gamma} \mathbf{n}^{\Gamma}(\mathbf{x}), \boldsymbol{\sigma}^{\Gamma}=\text { const., } \mathbf{x} \in \Gamma,
\end{aligned}
$$

where $\varepsilon^{\Gamma}$ and $\sigma^{\Gamma}$ are the macroscopic strain and stress tensors, which are the given constant symmetric tensors.

\subsection{Statistical description of random structure composites}

It is assumed that the representative macrodomain $w$ contains a statistically large number of realizations $\alpha$ of inclusions $v_{i} \in v^{(1)}(i=1,2, \ldots)$ of the constituent $v^{(1)}$ (providing validity of the standard probability theory technique). A random parameter $\alpha$ belongs to a sample space $\mathcal{A}$, over which a probability density $p(\alpha)$ is defined (see, e.g., Willis [1]). For any given $\alpha$, any random function $\mathbf{g}(\mathbf{x}, \alpha)$ (e.g., $\mathbf{g}=V, \boldsymbol{\sigma}, \boldsymbol{\varepsilon})$ is defined explicitly as one particular member, with label $\alpha$, of an ensemble realization. Then, the mean, statistical or ensemble average is defined by the angle brackets enclosing the quantity $\mathbf{g}$

$$
\langle\mathbf{g}\rangle(\mathbf{x})=\int_{\mathcal{A}} \mathbf{g}(\mathbf{x}, \alpha) p(\alpha) d \alpha .
$$

No confusion will arise below in notation of the random quantity $\mathbf{g}(\mathbf{x}, \alpha)$ if the label $\alpha$ is dropped for compactness of expressions unless such indication is necessary. One treats two material length scales (see, e.g., Torquato [4]): the macroscopic scale $L$, characterizing the extent of $w$, and the microscopic scale $a$, related with the heterogeneities $v_{i}$. Moreover, one supposes that applied field varies on a characteristic length scale $\Lambda$. The limit of our interests for both the material scales and field one is presented in an asymptotic sense

$$
L \gg \Lambda \geq a,
$$

as the scale of microstructure $a$ relative to the macroscale $L$ tends to zero. All the random quantities under discussion are described by statistically homogeneous random fields. For the alternative description of the random structure of a com165 posite material let us introduce a conditional probability density $\varphi\left(v_{i}, \mathbf{x}_{i} \mid v_{1}, \mathbf{x}_{1}\right)$, which is a probability density to find the $i$-th heterogeneity with the center $\mathbf{x}_{i}$ 
in the domain $v_{i}$ with fixed heterogeneity $v_{1}$ with the centers $\mathbf{x}_{1}$. The notation $\varphi\left(v_{i}, \mathbf{x}_{i} \mid ; v_{1}, \mathbf{x}_{1}\right)$ denotes the case $\mathbf{x}_{i} \neq \mathbf{x}_{1}$.

To prevent overlapping of different inclusions $\varphi\left(v_{i}, \mathbf{x}_{i} \mid ; v_{m}, \mathbf{x}_{m}\right)=0$ for values of $\mathbf{x}_{i}$ lying inside the "excluded volumes" $\cup v_{m i}^{0}$, where $v_{m i}^{0} \supset v_{m}$ with indicator function $V_{m i}^{0}$ is the "excluded volumes" of $\mathbf{x}_{i}$ with respect to $v_{m}$ (it is usually assumed that $\left.v_{m i}^{0} \equiv v_{m}^{0}\right)$, and $\varphi\left(v_{i}, \mathbf{x}_{i} \mid ; v_{m}, \mathbf{x}_{m}\right) \rightarrow \varphi\left(v_{i}, \mathbf{x}_{i}\right)$ as $\left|\mathbf{x}_{i}-\mathbf{x}_{m}\right| \rightarrow \infty$ (since no long-range order is assumed). $\varphi\left(v_{i}, \mathbf{x}\right)$ is a number density, $n(\mathbf{x})=n$ of component $v \ni v_{i}$ at the point $\mathbf{x}$ and $c^{(1)}$ is the concentration, i.e. volume 175 fraction, of the component $v_{i} \in v: c^{(1)}=\langle V\rangle=\bar{v}_{i} n, \bar{v}_{i}=\operatorname{mes} v_{i}(i=1,2, \ldots)$, $c^{(0)}(\mathbf{x})=1-\langle V\rangle$. Hereafter, if the pair distribution function $g\left(\mathbf{x}_{i}-\mathbf{x}_{m}\right) \equiv$ $\varphi\left(v_{i}, \mathbf{x}_{i} \mid ; v_{m}, \mathbf{x}_{m}\right) / n^{(k)}$ depends on $\mathbf{x}_{m}-\mathbf{x}_{i}$ only through $\left|\mathbf{x}_{m}-\mathbf{x}_{i}\right|$ it is called the radial distribution function (RDF, see for references and details Buryachenko et al. [30]). The notations $\langle()\rangle.(\mathbf{x})$ and $\left\langle() \mid. v_{1}, \mathbf{x}_{1}\right\rangle(\mathbf{x})$ will be used for the average and for the conditional average taken for the ensemble of a statistically homogeneous field $X=\left(v_{i}\right)$ at the point $\mathbf{x}$, on the condition that there are inclusions at the points $\mathbf{x}_{1}$. The notation $\langle(\cdot)\rangle_{i}(\mathbf{x})$ at $\mathbf{x} \in v_{i}$ means the statistical average over an ensemble realization of surrounding inclusions at the fixed $v_{i}$ whereas $\langle(\cdot)\rangle_{(i)}$ indicates the volume average over an inclusion $v_{i}$ in a single realization and $\langle(.)\rangle_{i} \equiv\left\langle\langle(.)\rangle_{(i)}\right\rangle$.

\subsection{Effective properties}

For statistically homogeneous media and homogeneous boundary conditions, the effective stiffness $\mathbf{L} *$ and effective compliance $\mathbf{M}$ governed by the expectation of Eqs. (2)

$$
\langle\boldsymbol{\sigma}\rangle=\mathbf{L}^{*}\langle\varepsilon\rangle, \quad\langle\varepsilon\rangle=\mathbf{M}^{*}\langle\boldsymbol{\sigma}\rangle
$$

are defined by general relations

$$
\mathbf{L}^{*}=\left\langle\mathbf{L} \mathbf{A}^{*}\right\rangle, \quad \mathbf{M}^{*}=\left\langle\mathbf{M B} \mathbf{B}^{*}\right\rangle,
$$

where $\mathbf{A}^{*}(\mathbf{x})$ and $\mathbf{B}^{*}(\mathbf{x})$ are the local strain and stress concentrator factors, respectively, obtained under pure mechanical loading $\varepsilon(\mathbf{x})=\mathbf{A}^{*}(\mathbf{x}) \varepsilon^{\Gamma}(9)$ and $\boldsymbol{\sigma}(\mathbf{x})=\mathbf{B}^{*}(\mathbf{x}) \boldsymbol{\sigma}^{\Gamma}$ (10), respectively. By the use of the decomposition of the material tensor $\mathbf{g}(\mathbf{g}=\mathbf{L}, \mathbf{M})$ Eqs. (14) can be rewritten in an equivalent form

$$
\mathbf{L}^{*}=\mathbf{L}^{(0)}+\left\langle\mathbf{L}_{1} \mathbf{A}^{*} V\right\rangle, \quad \mathbf{M}^{*}=\mathbf{M}^{(0)}+\left\langle\mathbf{M}_{1} \mathbf{B}^{*} V\right\rangle .
$$

The effective properties evaluations (15) are based on the estimations of the averages of polarisation tensors $\boldsymbol{\tau}\left(5_{1}\right)$ and $\boldsymbol{\eta}\left(5_{2}\right)$ averaged over the inclusion volume $v$. However, the representations $\boldsymbol{\tau}\left(6_{1}\right)$ and $\boldsymbol{\eta}\left(6_{2}\right)$ allows one to use only surface averages of these tensors. Namely, the volume averages of the strains and stresses inside inclusions can be expressed through the averages over the inclusion boundaries by the use of the Gauss's theorem

$$
\begin{aligned}
\langle\varepsilon V\rangle & =\frac{1}{2 \bar{v}} \int_{\Gamma}[\mathbf{u}(\mathbf{s}) \otimes \mathbf{n}(\mathbf{s})+\mathbf{n}(\mathbf{s}) \otimes \mathbf{u}(\mathbf{s})] d \mathbf{s}, \\
\langle\boldsymbol{\sigma} V\rangle & =\frac{1}{2 \bar{v}} \int_{\Gamma}[\mathbf{t}(\mathbf{s}) \otimes \mathbf{s}+\mathbf{s} \otimes \mathbf{t}(\mathbf{s})] d \mathbf{s}
\end{aligned}
$$


where in Eq. (17) one used the equality $\nabla(\mathbf{s} \otimes \boldsymbol{\sigma})=\boldsymbol{\sigma}$. Then the effective properties (15) can be expressed through the boundary integrals

$$
\begin{aligned}
\mathbf{L}^{*} & =\mathbf{L}^{(0)}+\mathbf{S}^{\epsilon *} \\
\mathbf{S}^{\epsilon *}\langle\varepsilon\rangle: & =\frac{1}{2 \bar{v}} \int_{\Gamma}\left\{\mathbf{t}(\mathbf{s}) \otimes \mathbf{s}+\mathbf{s} \otimes \mathbf{t}(\mathbf{s})-2 \mathbf{L}^{(0)}[\mathbf{u}(\mathbf{s}) \otimes \mathbf{n}(\mathbf{s})]\right\} d \mathbf{s}, \\
\mathbf{M}^{*} & =\mathbf{M}^{(0)}+\mathbf{S}^{\sigma *} \\
\mathbf{S}^{\sigma *}\langle\boldsymbol{\sigma}\rangle: & =\frac{1}{2 \bar{v}} \int_{\Gamma}\left\{\mathbf{u}(\mathbf{s}) \otimes \mathbf{n}(\mathbf{s})+\mathbf{n}(\mathbf{s}) \otimes \mathbf{u}(\mathbf{s})-2 \mathbf{M}^{(0)}[\mathbf{s} \otimes \mathbf{t}(\mathbf{s})]\right\} d \mathbf{s},
\end{aligned}
$$

195

a boundary conditions (9) and (10), respectively. The representation (18) for a particular case of the isotropic matrix is reduced to the corresponding equations proposed in [31, 32].

\section{Method of Fundamental Solutions (MFS)}

\subsection{The scheme of the Method of Fundamental Solutions (MFS)}

Estimation of the effective properties (18) and (19) through the boundary integrals over the inclusion surfaces $\Gamma_{i}$ is performed by substitutions of the corresponding integrals for a single representative inclusion $v_{i}$ into one or another micromechanical model (some of them will be considered in Section 4). Although an estimation of the boundary integrals for a single particle $v_{i}$ can be performed by the different BEMs, we consider the Method of Fundamental Solutions (MFS) which is a straightforward method that allows to approach the solution of Eqs. (1)-(3) for the known fundamental solutions. The solution is treated as a series of the fundamental solutions with singularities located outside the computational domain of the problem under consideration. The unknown coefficients of the series of the fundamental solutions are regarded as the strengths or the densities of the corresponding fundamental solutions. Namely, let the domains $v_{i}$ and $\hat{v}_{i}^{(1)}$ be open subsets in $R^{d}$ separated by the smooth boundary $\Gamma_{i}$ (see, e.g., [33]). We say that $\hat{v}_{i}^{(1)}$ with the smooth pseudoboundary $\hat{\Gamma}_{i}^{(1)}:=\partial \hat{v}_{i}^{(1)}$ embraces $v_{i}$ if $\left(v_{i} \cup \Gamma_{i}\right) \subset \hat{v}_{i}^{(1)}$. In a similar manner, one 215 can define a pseudo-boundary $\hat{\Gamma}_{i}^{(0)}:=\partial \hat{v}_{i}^{(0)}$ of an open subset $\hat{v}_{i}^{(0)}$ embracing the open domain $R^{d} \backslash\left(v_{i} \cup \Gamma_{i}\right)$. So, the grey regions in Figs. 1 and 2 are the inclusion $v_{i}$ with the boundary $\Gamma_{i}=\partial v_{i}$. The pseudo-boundaries $\hat{\Gamma}_{i}^{(0)}$ are constructed from $\Gamma_{i}=\partial v_{i}$ by a homothety transformation (26) (Fig. 1a) and by a normal direction choice for the source points (27) (Fig. 1b), respectively, which will be considered later.

One introduces the infinite body Green's function $\mathbf{G}^{(k)}$ of the Navier equation with homogeneous elastic modulus tensor $\mathbf{L}^{(k)}$, defined by

$$
\nabla\left\{\mathbf{L}^{(k)}\left[\nabla \otimes \mathbf{G}^{(k)}(\mathbf{x})\right]\right\}=-\boldsymbol{\delta} \delta(\mathbf{x}),
$$

of order $O\left(\int|\mathbf{x}|^{1-d} d|\mathbf{x}|\right)$ as $|\mathbf{x}| \rightarrow \infty$ and vanishing at infinity $(|\mathbf{x}| \rightarrow \infty)$ in 3 -D. We will also use the tensors of the fundamental stresses, tractions, and 
strains

$$
\begin{aligned}
\mathbf{D}^{(m)}(\mathbf{x}-\mathbf{s}) & =\mathbf{L}^{(m)} \nabla \mathbf{G}^{(m)}(\mathbf{x}-\mathbf{s}) \\
\mathbf{T}^{(m)}(\mathbf{x}, \mathbf{s}) & =-\mathbf{n}^{\Gamma}(\mathbf{x}) \mathbf{D}^{(m)}(\mathbf{x}-\mathbf{s}) \\
\mathcal{E}^{(m)}(\mathbf{x}-\mathbf{s}) & =\nabla \mathbf{G}^{(m)}(\mathbf{x}-\mathbf{s})
\end{aligned}
$$

In particular, for isotropic medium we reproduce the compact representation of 225 the mentioned Green functions valid for both 2-D and 3-D cases (see e.g. [4, 34]) in Appendix.

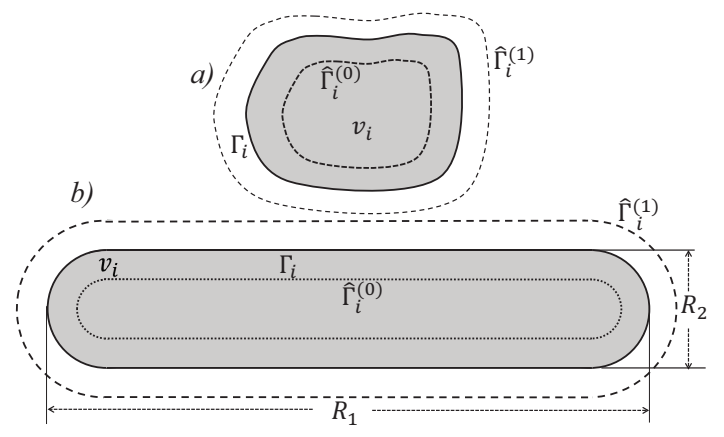

Fig. 1: The boundary $\Gamma_{i}=\partial v_{i}$ and the pseudo-boundaries $\hat{\Gamma}_{i}^{(0)}$ and $\hat{\Gamma}_{i}^{(1)}$ constructed by Eqs.

(26) (1a) and (27) (1b), which will be considered later.

We place $m$ points of the admissible singularities (or sources) $\left\{\mathbf{y}_{p}^{k}\right\}_{p=1}^{m}$ corresponding to the external $(k=0)$ and internal $(k=1)$ problems on the prescribed pseudoboundary surfaces $\hat{\Gamma}_{i}^{(0)}$ and $\hat{\Gamma}_{i}^{(1)}$, respectively. A slight modification of Alves and Silvestre [35] (see also [36]) results shows that it is possible to approach the boundary conditions for the displacement approximated by linear combinations of the corresponding fundamental solutions. Let the effective field $\overline{\mathbf{u}}(\mathbf{x})$ be prescribed in the collocation points $\left\{\mathbf{x}_{j}\right\}_{j=1}^{m} \in \Gamma_{i}$. Then the strength of FSs $\mathbf{f}_{i}^{(k)}=\left(\mathbf{f}_{i p \mid 1}^{(k)}, \ldots, \mathbf{f}_{i p \mid d}^{(k)}\right)^{\top}(p=1, \ldots, m)$ hold a $d m \times d m$ linear system of equations $(j=1, \ldots, d, l=1, \ldots, m)$

$$
\sum_{p=1}^{m} \sum_{q=1}^{d} f_{i p q}^{(1)} G_{j q}^{(1)}\left(\mathbf{r}_{l p}^{1}\right)=\sum_{p=1}^{m} \sum_{q=1}^{d} f_{i p q}^{(0)} G_{j q}^{(0)}\left(\mathbf{r}_{l p}^{0}\right)+\bar{u}_{j}\left(\mathbf{x}_{l}\right)
$$

where $\mathbf{r}_{l p}^{k}=\left(\mathbf{x}_{l}-\mathbf{y}_{p}^{k}\right)$ and $\left|\mathbf{r}_{l p}^{k}\right|$ is the distance between the collocation point (the field point), $\mathbf{x}_{l}$, and the location of the FSs (the source point), $\mathbf{y}_{p}^{k} \in \hat{\Gamma}_{i}^{(k)}$ $(k=0,1)$. In a similar manner, the traction boundary conditions $\left(7_{2}\right)$ can be obtained directly by taking summation of the traction FSs with the same strength $\mathbf{f}_{i}^{(k)}=\left(\mathbf{f}_{i p \mid 1}^{(k)}, \ldots, \mathbf{f}_{i p \mid d}^{(k)}\right)^{\top}(22)$ over the source points $\mathbf{y}_{p}^{k} \in \hat{\Gamma}_{i}^{(k)}$ outside 
the boundary $\Gamma_{i}(j, q=1, \ldots, d ; p=1, \ldots, m ; k=0,1)$ :

$$
\sum_{p=1}^{m} \sum_{q=1}^{d} f_{i p q}^{(1)} T_{j q}^{(1)}\left(\mathbf{r}_{l p}^{1}\right)=\sum_{p=1}^{m} \sum_{q=1}^{d} f_{i p q}^{(0)} T_{j q}^{(0)}\left(\mathbf{r}_{l p}^{0}\right)+\bar{t}_{j}\left(\mathbf{x}_{l}\right),
$$

where $\overline{\mathbf{t}}=\overline{\boldsymbol{\sigma}} \mathbf{n}^{\Gamma_{i}}\left(\mathbf{x}_{l}\right)$. We assumed in the system (22) and (23) that the numbers of both the field points $\left\{\mathbf{x}_{l}\right\}_{l=1}^{m}$ and source ones $\left\{\mathbf{y}_{p}^{k}\right\}_{p=1}^{m}(k=0,1)$ coincide. In such a case the strength of the FSs, $\mathbf{f}_{i}$, are determined uniquely, provided that the corresponding coefficient matrix is nonsingular. If $\overline{\mathbf{u}}(\mathbf{x})$ is prescribed in more points $\mathbf{x}_{1}, \ldots, \mathbf{x}_{n} \in \Gamma_{i}$, with $n>m$, the system (22) and (23) is overdetermined, and the strength can be chosen by the discrete least-squares method (see [33, $35])$ which is not considered in more details in the current paper.

Once the strength of the FS, $\mathbf{f}_{i}^{(k)}$, are found by solving the system (22) and (23) at $\left\{\mathbf{x}_{l}\right\}_{l=1}^{m} \in \Gamma_{i}$, the displacement vector in both the inclusion $v_{i}$ and matrix $R^{d} \backslash v_{i}$ is then calculated straightforwardly by using the left-right side and right-hand side, respectively, of Eq. (22) with values of replaced radius-vectors $\mathbf{x}_{l} \rightarrow \mathbf{x}_{s}$ corresponding to location of a point $\mathbf{x}_{s}$. All other field variables can be obtained directly by taking summation of the same strength $\mathbf{f}_{i}^{(k)}(22)$ and (23) of the corresponding FSs over the source points $\mathbf{y}_{p}^{k} \in \hat{\Gamma}_{i}^{(k)}(k=0,1)$ outside the boundary $\Gamma_{i}$, e.g. the displacement and stress induced by $m$ FSs can be estimated by the equations $(j, l=1, \ldots, d)$ :

$$
\begin{aligned}
u_{j}\left(\mathbf{x}_{s}\right) & =\sum_{p=1}^{m} \sum_{q=1}^{d} f_{i p q}^{(k)} G_{j q}^{(k)}\left(\mathbf{r}_{s p}^{k}\right)+\bar{u}_{j}\left(\mathbf{x}_{s}\right) \delta_{k 0}, \\
\sigma_{j l}\left(\mathbf{x}_{s}\right) & =\sum_{p=1}^{m} \sum_{q=1}^{d} f_{i p q}^{(k)} D_{j l q}^{(k)}\left(\mathbf{r}_{s p}^{k}\right)+\bar{\sigma}_{j l}\left(\mathbf{x}_{s}\right) \delta_{k 0}
\end{aligned}
$$

for $\mathbf{x}_{s} \in v_{i}(k=1)$ and $\mathbf{x}_{s} \in\left(R^{d} \backslash v_{i}\right)(k=0)$, respectively.

A specific feature of the MFS is some freedom in choosing the source points with respect to the accuracy of the numerical solution. Young et al. [37] estimated an excellent accuracy of the MFS for the positions of the source points $\mathbf{y}$ in computational domain through the field points $\mathbf{x}_{l}(l=1, \ldots, m)$ by a homothety transformation:

$$
\mathbf{y}_{l}=\mathbf{x}_{i}+b\left(\mathbf{x}_{l}-\mathbf{x}_{i}\right),
$$

where $\mathbf{x}_{i}$ is a homothetic center coinciding with the particle center. Once the parameters $0<b<1$ and $1<b$ for $k=1$ and $k=0$, respectively, are chosen, the distribution of the source points is determined. The pceudo-boundaries $\hat{\Gamma}_{i}^{(0)}$ and $\hat{\Gamma}_{i}^{(1)}$ in Fig. 1a are obtained from the boundary $\hat{\Gamma}_{i}$ of the grey inclusion $v_{i}$ by the homothety transformation (26).

However, for the thin inclusions with the thickness $2 h$ (e.g. for the tri-axial ellipsoid with distinct semi-axis lengths $\left.a_{1}>a_{2} \gg a_{3}, h=a_{3}\right)$ the distances $D\left(\mathbf{y}_{l}, \Gamma_{i}\right)$ (defining the matrix coefficients $G_{j q}^{(k)}\left(\mathbf{r}_{l p}^{1}\right)(22)$ and $T_{j q}^{(k)}\left(\mathbf{r}_{l p}^{1}\right)(23)$ are 
essentially differ one from another points $\mathbf{x}_{i}$. Here $D\left(\mathbf{y}_{l}, \Gamma_{i}\right)$ denotes the Euclidean distance between $\mathbf{y}_{l}$ and the boundary of $v_{i}$, or in other words, the radius of the largest inscribed disk with center $\mathbf{y}_{l}$. Because of this, for the thin inclusions the following allocations of the source points $(k=0,1)$

$$
\mathbf{y}_{l}^{k}=\mathbf{x}_{l}+(2 k-1) d_{k} \mathbf{n}^{\Gamma_{i}}\left(\mathbf{x}_{l}\right)
$$

250

with $d=D\left(\mathbf{y}_{l}, \Gamma_{i}\right)=10-30 \% h$ is preferable.

The algorithm of the normal direction choice for the source points (27) proposed by Alves and Antunes [38] was accomplished by so called glocal choice for the coefficient $d_{k}(k=0,1)$ recently presented in [39]. A simplest choice of a constant $d_{k}$ is a non-optimal one that works in most cases. In such a case, the pseudo-boundaries $\hat{\Gamma}^{(1)}$ and $\hat{\Gamma}^{(0)}$ can be presented as the boundaries of Minkowski addition and subtraction

$$
\hat{v}^{(1)}=v_{i} \oplus\left(\mathbf{0}, d_{0}\right), \quad \hat{v}^{(0)}=v_{i} \ominus b\left(\mathbf{0}, d_{1}\right)
$$

, respectively, of the domain $v_{i}$, where $b\left(\mathbf{0}, d_{k}\right)$ stands the ball of radius $d$ centered at the origin $\mathbf{0}$. As an example, a grey cross-section of a spherocylinder (or a capsule) with the aspect ratio $\alpha=R / h$ is presented in Fig. 1b. The pseudo-boundaries $\hat{\Gamma}_{i}^{(0)}$ and $\hat{\Gamma}_{i}^{(1)}$ in Fig. $1 \mathrm{~b}$ are obtained from the boundary

${ }_{255} \hat{\Gamma}_{i}$ of the grey inclusion $v_{i}$ by the normal direction choice for the source points (27) with $d_{1}=h / 2$ and $d_{0}=h / 2$, respectively. In so doing, the aspect ratios of the domains with the pseudo-boundaries $\hat{\Gamma}_{i}^{(1)}$ and $\hat{\Gamma}_{i}^{(0)}$ are $(2 \alpha+1) / 3$ and $(2 \alpha-1)$ while the aspect ratios of the corresponding domains obtained by the homothety transform (27) do not change and equal $\alpha$.

Alves [39] (see also [38]) analysed optimisation of more general stresslet allocation techniques for convex and concave regions. However, more detail consideration of optimization of FS's allocation substantially reducing computational time for the elastic problem solution is beyond the scope of the current study.

\subsection{The matrix representation of the MFS}

Construction and solution of the linear algebraic system (22) is straightforward (see, e.g., $[37,40])$. However, we consider it in more details for the $2 \mathrm{D}$ domain $v_{i}$. The singularities $Y_{p}^{0}=\left(y_{p \mid 1}^{0}, y_{p \mid 2}^{0}\right)$ and $Y_{p}^{1}=\left(y_{p \mid 1}^{1}, y_{p \mid 2}^{1}\right)(p=1, \ldots, P)$ are fixed on the boundaries $\hat{\Gamma}_{i}^{(0)}$ of $\hat{v}_{i}^{(0)}$ and $\hat{\Gamma}_{i}^{(1)}$ of $\hat{v}_{i}^{(1)}$, respectively. A set of $P$ collocation points $X_{p}=\left(x_{p \mid 1}, x_{p \mid 2}\right)(p=1, \ldots, P)$ is chosen on $\Gamma_{i}$. Then the system (22) can be presented in the matrix form

$$
\mathbf{A f}=\left(\begin{array}{cccc}
\mathbf{A}_{11} & \mathbf{A}_{1,2} & \ldots & \mathbf{A}_{1, P} \\
\mathbf{A}_{2,1} & \mathbf{A}_{2,2} & \ldots & \mathbf{A}_{2, P} \\
\vdots & \vdots & \ddots & \vdots \\
\mathbf{A}_{P, 1} & \mathbf{A}_{P, 2} & \ldots & \mathbf{A}_{P, P}
\end{array}\right)\left(\begin{array}{c}
\mathbf{f}_{1} \\
\mathbf{f}_{2} \\
\vdots \\
\mathbf{f}_{P}
\end{array}\right)=\left(\begin{array}{c}
\mathbf{g}_{1} \\
\mathbf{g}_{2} \\
\vdots \\
\mathbf{g}_{P}
\end{array}\right)=\mathbf{g}
$$

where the $4 P \times 4 P$ matrix $\mathbf{A}$ with the submatrices

$$
\mathbf{A}_{\alpha, \beta}=\left(\begin{array}{ll}
\mathbf{A}_{\alpha, \beta}^{1,1} & \mathbf{A}_{\alpha, \beta}^{1,2} \\
\mathbf{A}_{\alpha, \beta}^{2,1} & \mathbf{A}_{\alpha, \beta}^{2,2}
\end{array}\right)
$$


and

$$
\begin{aligned}
\mathbf{f}_{p} & =\left(\mathbf{f}_{p}^{(0)}, \mathbf{f}_{p}^{(1)}\right)^{\top}=\left(f_{p \mid 1}^{(0)}, f_{p \mid 2}^{(0)}, f_{p \mid 1}^{(1)}, f_{p \mid 2}^{(1)}\right)^{\top} \in R^{4}, \\
\mathbf{g}_{p} & =\left(\mathbf{g}_{p}^{(0)}, \mathbf{g}_{p}^{(1)}\right)^{\top}=\left(g_{p \mid 1}^{(0)}, g_{p \mid 2}^{(0)}, g_{p \mid 1}^{(1)}, g_{p \mid 2}^{(1)}\right)^{\top} \in R^{4},
\end{aligned}
$$

where $f_{p \mid j}^{(\delta)}=f_{p \mid j}^{(\delta)}\left(Y_{p}^{\delta}\right), g_{p \mid j}^{(\delta)}=\bar{u}_{p \mid j} \delta_{0 \delta}+\bar{t}_{p \mid j} \delta_{1 \delta}$, and $j=1,2 ; p, \alpha, \beta=1, \ldots, P ; \delta=$ 0,1 . It is observed for $d=2,3$ that accurate numerical results could be obtained when the source points for the pseudoboundary $\hat{\Gamma}_{i}^{(0)}$ of the submerged stresslets are located off the boundary at about $70-90 \%$ (26) of the distance from the center (see for details Young et al. [37].

It should be mentioned that for two limiting cases of the rigid inclusions and pores, one can consider one pseudo-boundary $\hat{\Gamma}_{i}^{(0)}$ instead of two pseudoboundaries $\hat{\Gamma}_{i}^{(0)}$ and $\hat{\Gamma}_{i}^{(1)}$. Namely, the matrix Eq. (28) is formally kept without changes. However, now the matrix $\mathbf{A}$ has a dimension $2 P \times 2 P$ and for the rigid inclusions the submatrices

$$
\mathbf{A}_{\alpha, \beta}=\mathbf{A}_{\alpha, \beta}^{1,1}
$$

are defined by the displacement fundamental tensor

$$
\mathbf{A}_{\alpha, \beta}^{1,1}=-\mathbf{G}^{(0)}\left(\left|X_{\alpha}-Y_{0}^{\delta}\right|\right) \in R^{2 \times 2},
$$

and

$$
\mathbf{f}_{p}=\left(f_{p \mid 1}^{(0)}, f_{p \mid 2}^{(0)}\right)^{\top} \in R^{2}, \quad \mathbf{g}_{p}=\left(g_{p \mid 1}, g_{p \mid 2}\right)^{\top} \in R^{2},
$$

where $f_{p \mid j}^{(0)}=f_{p \mid j}^{(0)}\left(Y_{p}^{0}\right), g_{p \mid j}=\bar{u}_{p \mid j}$, and $j=1,2 ; p, \alpha, \beta=1, \ldots, P$. In the case of replacement of rigid inclusions by the porous, the equations (34)-(36) should be also replaced by the following equations

$$
\begin{gathered}
\mathbf{A}_{\alpha, \beta}=\mathbf{A}_{\alpha, \beta}^{2,1} \\
\mathbf{A}_{\alpha, \beta}^{2,1}=-\mathbf{T}^{(0)}\left(\left|X_{\alpha}-Y_{0}^{\delta}\right|\right) \in R^{2 \times 2}, \\
\mathbf{f}_{p}=\left(f_{p \mid 1}^{(0)}, f_{p \mid 2}^{(0)}\right)^{\top} \in R^{2}, \quad \mathbf{g}_{p}=\left(g_{p \mid 1}, g_{p \mid 2}\right)^{\top} \in R^{2},
\end{gathered}
$$

respectively, where $f_{p \mid j}^{(0)}=f_{p \mid j}^{(0)}\left(Y_{p}^{0}\right), g_{p \mid j}=\bar{t}_{p \mid j}$, and $j=1,2 ; p, \alpha, \beta=1, \ldots, P$.

However, the MFS coefficient matrix A (29) (as well as (34) and (37)) of the system (22) and (23) is often severely ill-conditioned and the system of linear algebraic equations (22) and (23) cannot be solved by direct methods, such as, 
e.g., the least-squares method, producing a highly unstable solution which increases dramatically as the number of boundary collocation points $m$ increases. Several regularization procedures have been proposed to remedy the instability and accuracy loss in the solution of such ill-conditioned matrix equations (see, e.g., [41]). However, we only consider the Tikhonov regularization (TR) method, see e.g., Tikhonov and Arsenin [22], since it is simple, noniterative, and it provides an explicit solution. Namely, the TR procedure consists of seeking a solution which minimizes a criterion made up of the sum of two summands. The first one is a possibly weighted least square term while the second one is a quadratic penalty term on the solution, that is

$$
\min \left\{\|\mathbf{A f}-\mathbf{g}\|_{2}^{2}+\lambda\left\|\mathbf{I}^{\mathrm{dm}} \mathbf{f}\right\|_{2}^{2}\right\},
$$

where $\|(\cdot)\|_{2}$ denotes the Euclidean norm while for the zeroth order TR method, $\mathbf{I}^{\mathrm{dm}}=\operatorname{diag}(1, \ldots, 1) \in R^{d m \times d m}$ is the identity matrix. Formally, the TR procedure provides an explicit solution $\mathbf{f}_{\lambda}$ of the regularized system of equations,

$$
\left(\mathbf{A}^{\top} \mathbf{A}+\lambda \mathbf{I}^{\mathrm{dm}}\right) \mathbf{f}=\mathbf{A}^{\top} \mathbf{g} .
$$

The regularization parameter $\lambda \geq 0$ often controls the relative weight of the penalty term in the criterion. For the parameter $\lambda=0$, Eq. (41) degenerates to the solution of the least squares problem which is completely dominated by contributions from data errors and rounding errors. By adding the penalty term, we are able to reduce these contributions and maintain the penalty term to be of reasonable size. However, the optimal determination of a regularization parameter $\lambda$ is essential for the effectiveness of the TR method applied to the illconditioned interpolation matrix equation. In the L-curve criterion, the optimal value $\lambda$ is intuitively chosen at the "corner" of the L-curve defined as

$$
L=\left\{\log \left\|\mathbf{A \mathbf { f } _ { \lambda }}-\mathbf{g}\right\|_{2}, \log \left\|\mathbf{I \mathbf { f } _ { \lambda }}\right\|_{2}, \lambda \geq 0\right\}
$$




\section{Effective elastic moduli of composite materials}

\subsection{Some classical hypotheses}

The basic hypotheses of micromechanics are well known and presented in the form adopted to the use of the volume integral over the inclusion volume (see, 290 e.g., [6]). We present in this Subsection the same hypotheses in the form which are most adopted for the subsequent application of the boundary integrals over the inclusion boundaries. Namely, we now apply so-called effective field hypothesis $(\mathrm{EFH})$ which is the main approximate hypothesis of many micromechanical methods:

Hypothesis H1. Each heterogeneity $v_{i}$ is located in the field $(\boldsymbol{\vartheta}=\boldsymbol{\varepsilon}, \boldsymbol{\sigma})$

$$
\overline{\boldsymbol{\vartheta}}_{i}(\mathbf{y}) \equiv \overline{\boldsymbol{\vartheta}}\left(\mathbf{x}_{i}\right)\left(\mathbf{y} \in v_{i}\right),
$$

which is homogeneous over the heterogeneity $v_{i}$.

For the boundary variables $\left(\mathbf{s} \in \Gamma_{i}\right)$, the hypothesis $\mathbf{H} \mathbf{1}$ can be presented in the local coordinate systems connected with $v_{i}$

$$
\overline{\mathbf{u}}_{i}(\mathbf{s}) \equiv \overline{\boldsymbol{\varepsilon}}\left(\mathbf{x}_{i}\right) \mathbf{s} \quad \overline{\mathbf{t}}_{i}(\mathbf{s}) \equiv \overline{\boldsymbol{\sigma}}\left(\mathbf{x}_{i}\right) \mathbf{n}^{\Gamma_{i}}(\mathbf{s}),
$$

where $\mathbf{n}^{\Gamma_{i}}(\mathbf{s})$ is unit outward normal at $\mathbf{s} \in \Gamma_{i}$.

According to hypothesis H1a and in view of the linearity of the problem there exist constant fourth and second-rank tensors $\mathbf{B}_{i}(\mathbf{x}), \mathbf{R}_{i}(\mathbf{x})$, such that

$$
\boldsymbol{\sigma}(\mathbf{x})=\mathbf{B}_{i}(\mathbf{x}) \overline{\boldsymbol{\sigma}}\left(\mathbf{x}_{i}\right), \quad \bar{v}_{i} \boldsymbol{\eta}(\mathbf{x})=\mathbf{R}_{i}(\mathbf{x}) \overline{\boldsymbol{\sigma}}\left(\mathbf{x}_{i}\right), \quad \mathbf{x} \in v_{i},
$$

where $v_{i} \subset v^{(1)}$ and $\mathbf{R}_{i}(\mathbf{x})=\bar{v}_{i} \mathbf{M}_{1}^{(1)}(\mathbf{x}) \mathbf{B}_{i}(\mathbf{x})$. According to Eshelby's [43] (see also [44]) theorem for the ellipsoidal inclusion $v_{i}$, there is the following relation between the averaged tensors (48) $\mathbf{R}_{i}=\bar{v}_{i} \mathbf{Q}_{i}^{-1}\left(\mathbf{I}-\mathbf{B}_{i}\right)$, where $\mathbf{g}_{i} \equiv$ $\langle\mathbf{g}(\mathbf{x})\rangle_{(i)} \quad\left(\mathbf{g}\right.$ stands for $\left.\mathbf{B}_{i}, \mathbf{R}_{i}\right)$, where $\mathbf{Q}_{i}=\mathbf{L}^{(0)}\left(\mathbf{I}-\mathbf{S}_{i}\right)$ ir represented through the Eshelby tensor $\mathbf{S}_{i}$. For example, for the homogeneous ellipsoidal domain $v_{i}$ we obtain $\left(\mathbf{x} \in v_{i}\right)$

$$
\mathbf{B}_{i}(\mathbf{x}) \equiv \mathbf{B}_{i}=\left(\mathbf{I}+\mathbf{Q}_{i} \mathbf{M}_{1}^{(i)}\right)^{-1} .
$$

In the general case of coated inclusions $v_{i}$, the tensors $\mathbf{B}_{i}(\mathbf{x})$ can be found by the transformation method by Dvorak and Benveniste [45] (see for references and details $[6])$.

Different methods can be employed to truncate the hierarchies of corresponding integral equations (see for references,e.g., [6]). Probably the most popular closing assumption is "quasi-crystalline approximation" by Lax [46] which neglects direct interaction between each pair of heterogeneities although such an interaction takes place though the effective field generated by all surrounding inclusions. 
Hypothesis H2, "quasi-crystalline" approximation. It is supposed that the mean value of the effective field at a point $\mathbf{x} \in v_{i}$ does not depend on the stress field inside surrounding heterogeneities $v_{j} \neq v_{i}\left(\mathbf{x} \in v_{i}, \mathbf{s} \in \Gamma_{i}\right)$ :

$$
\left\langle\overline{\boldsymbol{\vartheta}}_{i}(\mathbf{x}) \mid ; v_{j}, \mathbf{x}_{j}\right\rangle=\left\langle\overline{\boldsymbol{\vartheta}}_{i}\right\rangle, \quad\left\langle\bar{\chi}_{i}(\mathbf{s}) \mid ; v_{j}, \mathbf{x}_{j}\right\rangle=\left\langle\bar{\chi}_{i}\right\rangle .
$$

To make further progress, the hypothesis of "ellipsoidal symmetry" for the distribution of inclusions attributed to Willis [24] is widely used:

Hypothesis H3, "ellipsoidal symmetry". The conditional probability density function $\varphi\left(v_{j}, \mathbf{x}_{j} \mid ; v_{i}, \mathbf{x}_{i}\right)$ depends on $\mathbf{x}_{j}-\mathbf{x}_{i}$ only through the combination $\rho=\left|\left(\mathbf{a}_{i j}^{0}\right)^{-1}\left(\mathbf{x}_{j}-\mathbf{x}_{i}\right)\right|$ :

$$
\varphi\left(v_{j}, \mathbf{x}_{j} \mid ; v_{i}, \mathbf{x}_{i}\right)=h(\rho),
$$

where the matrix $\left(\mathbf{a}_{i j}^{0}\right)^{-1}$ (which is symmetric in the indexes $i$ and $j, \mathbf{a}_{i j}^{0}=\mathbf{a}_{j i}^{0}$ ) defines the ellipsoid excluded volume $v_{i j}^{0}=\left\{\mathbf{x}:\left|\left(\mathbf{a}_{i j}^{0}\right)^{-1} \mathbf{x}\right|^{2}<1\right\}$.

It is reasonable to assume that $\left(\mathbf{a}_{i j}^{0}\right)^{-1}$ identifies a matrix of affine transformation that transfers the ellipsoid $v_{i j}^{0}$ being the "excluded volume" ("correlation hole") into a unit sphere.

\subsection{Some popular methods}

Hypotheses H1-H3 allow one to obtain the explicit solution by the method of effective field (MEF, see for references and details [6]) for the stress distribution (the strain field can be considered in a similar manner) for identical aligned heterogeneities $\left(v_{i}=v_{j}=v_{1}, \mathbf{x} \in v_{1}\right)$

$$
\begin{aligned}
\langle\overline{\boldsymbol{\sigma}}\rangle_{1}(\mathbf{x}) & =\mathbf{Y}\langle\boldsymbol{\sigma}\rangle, \\
\langle\boldsymbol{\sigma}\rangle_{1}(\mathbf{x}) & =\mathbf{B}_{i}(\mathbf{x}) \mathbf{Y}\langle\boldsymbol{\sigma}\rangle, \\
\mathbf{M}^{*} & =\mathbf{M}^{(0)}+n^{(1)} \mathbf{R}_{i} \mathbf{Y}, \\
\mathbf{Y}^{-1} & =\mathbf{I}-n^{(1)} \mathbf{R}_{i} \mathbf{Q}_{i}^{0},
\end{aligned}
$$

where the matrix $\mathbf{Y}$ is determined by the action of the surrounding inclusions and for the sake of simplicity of the subsequent calculation it is usually assumed that the shape of "correlation hole" $v_{i j}^{0}$ does not depend on the inclusion $v_{j}$ : $v_{i j}^{0}=v_{i}^{0}$ and $\mathbf{Q}_{i j}^{0}=\mathbf{Q}_{i}^{0} \equiv \mathbf{Q}\left(v_{i}^{0}\right)$.

As pointed out by Benveniste [12], the essential assumption in the MoriTanaka [11] method (MTM) states that each inclusion $v_{i}$ behaves as an isolated one in the infinite matrix and subject to some effective stress field $\overline{\boldsymbol{\sigma}}_{i}$ coinciding with the average stress in the matrix

$$
\left\langle\overline{\boldsymbol{\sigma}}_{i}\right\rangle=\langle\boldsymbol{\sigma}\rangle^{(0)} .
$$

Using Eq. (56) as the closing assumption and substituting the hypothesis $\mathbf{H 2}$ leads to the next representation for both the statistical average local stresses 
and effective compliance

$$
\begin{aligned}
\langle\boldsymbol{\sigma}\rangle_{i}(\mathbf{x}) & =\mathbf{B}_{i}(\mathbf{x})\left[c^{(0)} \mathbf{I}+c^{(1)} \mathbf{B}_{i}\right]^{-1}\langle\boldsymbol{\sigma}\rangle \\
\mathbf{M}^{*} & =\mathbf{M}^{(0)}+c^{(1)} \mathbf{R}_{i}\left[c^{(0)} \mathbf{I}+c^{(1)} \mathbf{B}_{i}\right]^{-1} .
\end{aligned}
$$

325 For the identical ellipsoidal inhomogeneous heterogeneities $v_{i}$ homothetical to $v_{i}^{0}$, equivalences of Eqs. (53), (54) and (57), (58), respectively, are demonstrated in, e.g., [6]). However, the representations (53), (54) and (57), (58), respectively, do not coincide even for the identical aligned isotropic fibers if $v_{i}$ and $v_{i}^{0}$ are not homothetic (in particularly, if $v_{i}$ is not an ellipsoid, see [6]).

330 It should be mentioned that the effective compliances (54) and (58) are traditionally expressed through the averages of the tensors $\mathbf{B}_{i}(\mathbf{x})$ and $\mathbf{R}_{i}(\mathbf{x})$ over the inclusion volumes $v_{i}$. However, these tensors can be also estimated through the averages over the inclusion boundaries $\mathbf{s} \in \Gamma_{i}$ of the fields $\boldsymbol{\vartheta}(\mathbf{s})$ $(\boldsymbol{\vartheta}=\boldsymbol{\varepsilon}, \boldsymbol{\sigma})$ corresponding to the homogeneous loading $\overline{\boldsymbol{\sigma}} \equiv$ const. $(c \rightarrow 0)$ of a 335 single inclusion $v_{i}$ :

$$
\begin{aligned}
& \mathbf{B}_{i} \overline{\boldsymbol{\sigma}}=\frac{1}{2 \bar{v}_{i}} \int_{\Gamma_{i}}[\mathbf{t}(\mathbf{s}) \otimes \mathbf{s}+\mathbf{s} \otimes \mathbf{t}(\mathbf{s})] d \mathbf{s} \\
& \mathbf{R}_{i} \overline{\boldsymbol{\sigma}}=\frac{1}{2} \int_{\Gamma_{i}}\left\{\mathbf{u}(\mathbf{s}) \otimes \mathbf{n}(\mathbf{s})+\mathbf{n}(\mathbf{s}) \otimes \mathbf{u}(\mathbf{s})-2 \mathbf{M}^{(0)}[\mathbf{t}(\mathbf{s}) \otimes \mathbf{s}]\right\} d \mathbf{s} .
\end{aligned}
$$

In such a case the effective compliances $\mathbf{M}^{*}(54)$ and (58) are estimated through the boundary integrals as in Eqs. (59) and (60). In so doing, the stress concentration tensor $\mathbf{B}_{i}(\mathbf{x})$ in Eqs. (53) and (57) is estimated by Eq. (45) at $k=1$.

\section{Numerical results}

We consider the problem for one inclusion in the infinite homogeneous matrix with large elastic mismatch of the inclusion (the Young modulus $E^{(1)}=100$ and Poisson ratio $\left.\nu^{(1)}=0.49\right)$ and the matrix $\left(E^{(0)}=1, \nu^{(0)}=0.45\right)$. In order to estimate the approximations introduced by discretizations implemented in the MFS in Section 4.2 and for the evaluation of the solution (29) we compare numerical results with a reference analytical solution for a single circle inclusion inside the infinite matrix in a plane strain problem. In the following experiments (Fig. 2), we considered uniformly spaced collocation points on $\Gamma_{i}$ and the same number of source points on $\hat{\Gamma}_{i}^{(1)}$ and $\hat{\Gamma}_{i}^{(0)}$ when the source sets prescribed by Eqs. (26) and (27) coincide. We consider a uniform remote tension $\bar{\sigma}_{i j}=\delta_{i 1} \delta_{j 1}$ generating a homogeneous stress distribution inside circle inclusion $\boldsymbol{\sigma}(\mathbf{x})=\mathbf{B}_{i}(\mathbf{x}) \overline{\boldsymbol{\sigma}}\left(\mathbf{B}_{i}(\mathbf{x}) \equiv\right.$ const., $\left.\quad \mathbf{x} \in v_{i}\right)$ which is analytically estimated by means of the Eshelby tensor. The value $B_{i \mid 1111}(\mathbf{x}) \equiv 1.4587$ analytically evaluated is compared with their numerical values $B_{i \mid 1111}(\mathbf{x})$ esti-

355 mated by both the direct (least-squares) method of solution of Eq. (29) and the pseudo-inverse TR procedure (42) in the vicinity of the inclusion boundary $B_{i \mid 1111}(\mathbf{s}) \equiv \lim B_{i \mid 1111}(\mathbf{x})\left(\mathbf{x} \rightarrow \mathbf{s}, \mathbf{x} \in v_{i}, \mathbf{s} \in \Gamma_{i}\right)$ In Fig. 2 the curves 1 and 
2 are estimated by the direct method and the TR procedure, respectively, for $P=30, d=0.6(27)$, and $\lambda=10^{-5}$. The errors corresponding to the curves 1 and 2 equal $6.5 \%$ and $0.23 \%$, respectively. Increasing of the number of source points $P=60$ leads to increasing of accuracy of the TR procedure (42) (curve 4 with the error $0.00068 \%$ ) and to osculation of the direct solution (29) (curve 3, the error $35 \%$ ). Subsequent increasing of the number of source points $P=200$ with $d=0.1$ provide the error $0.000016 \%$ of the stress distribution $B_{i \mid 1111}(\mathbf{s})$ $\left(\mathbf{s} \in \Gamma_{i}\right)$ (42) while the approximative osculating solution (29) takes the negative values $\left(B_{i \mid 1111}(\mathbf{s})<0\right.$. at some $\left.\mathbf{x} \in \Gamma_{i}\right)$ and loses a physical meaning.

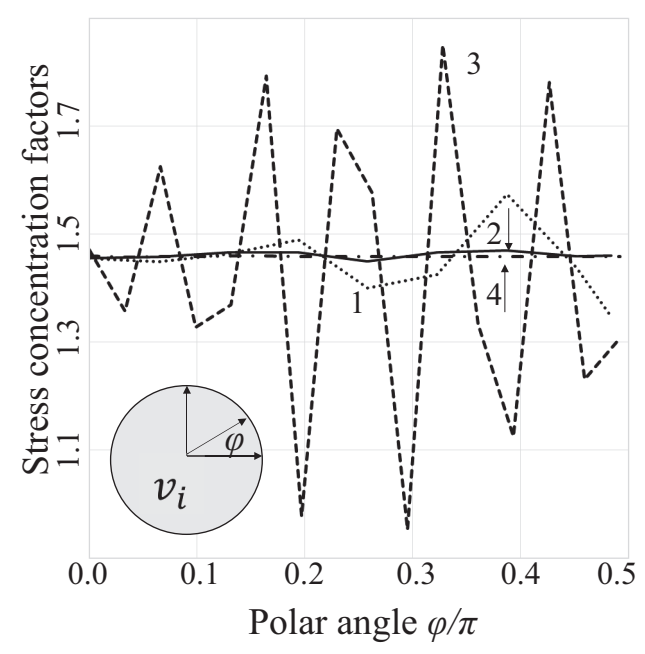

Fig. 2: Stress concentration factor $B_{i \mid 1111}(\mathbf{s})$ vs a polar angle $\phi / \pi$.

Now we compare the numerical solutions $B_{i \mid 1111}(\mathbf{x})\left(\mathbf{x} \in v_{i}\right)$ obtained by the TR procedure (42) for two types of source sets generated by both the homothety transformation (26) and the normal direction choice (27) with $P=375, b=$ $0.9, d=0.1 R_{2}, \lambda=10^{-5}$. For demonstration of the difference between the mentioned numerical results, we analyse the elliptic inclusion

$$
x_{1}^{2} / R_{1}^{2}+x_{2} / R_{2}^{2}=1
$$

with distinct semiaxis lengths $\alpha:=R_{2} / R_{1}=0.1,0.2,0.3$ corresponding to the analytical results $B_{i \mid 1111}(\mathbf{x}) \equiv 5,731,3.402,2.601\left(\mathbf{x} \in v_{i}, \mathbf{x}=\left(x_{1}, 0\right)^{\top}\right)$, 370 respectively. For $\alpha=0.3$ both the source sets (26) and (27) provide the comparable accuracies (the errors $0.28 \%$ and, $0.016 \%$ respectively). However, at the flattening of the elliptical inclusion (with decreasing of $\alpha$ from 0.3 to 0.1 ) demonstrates an advantage of the algorithm (27) with respect to the homothety transformation (26). Indeed, the errors of the TP procedure (42) exploiting 375 the source set (26) increases to $1.8 \%$ (Fig. 3, curve 1) and to $9.2 \%$ (curve 3 ) for $\alpha=0.2$ and $\alpha=0.1$, respectively, while the error of the same method (42) with the source set (27) leads to the errors $0.021 \%$ (curve 2) and $0.31 \%$ 
(curve 4), respectively. The reason of so much dramatic decline of accuracy of the estimations corresponding to the source set (26) is explained by an essentially different variation of the distances $D\left(\mathbf{y}_{l}, \Gamma_{i}\right)$ of the source points from the boundary $\Gamma_{i}$. For the normal direction choice for the source points (27), this distance $d=D\left(\mathbf{y}_{l}, \Gamma_{i}\right) \equiv 0.1 R_{2}$ is the constant for $\forall \mathbf{y}_{l} \in \hat{\Gamma}_{i}^{(k)}(k=0,1)$ while for homothety transformation (26), the distances $D\left(\mathbf{y}_{l}, \Gamma_{i}\right)$ equal $d_{2}=0.1 R_{2}$ (as in Eq. (26)) and $d_{1}=0.1 R_{1}=10 d_{2}$ for $\mathbf{y}_{l}=\left(0,0.9 R_{2}\right)^{\top}$ and $\mathbf{y}_{l}=\left(0.9 R_{1}, 0\right)^{\top}$, respectively. Thus, TR procedure (42) accompanied by the normal direction choice for the source points (27) provides an excellent accuracy of the solution of the elastic problem for a single inclusion $v_{i} \subset R^{2}$ in a wide range of both the elastic mismatch and the aspect ratio of the inclusion.

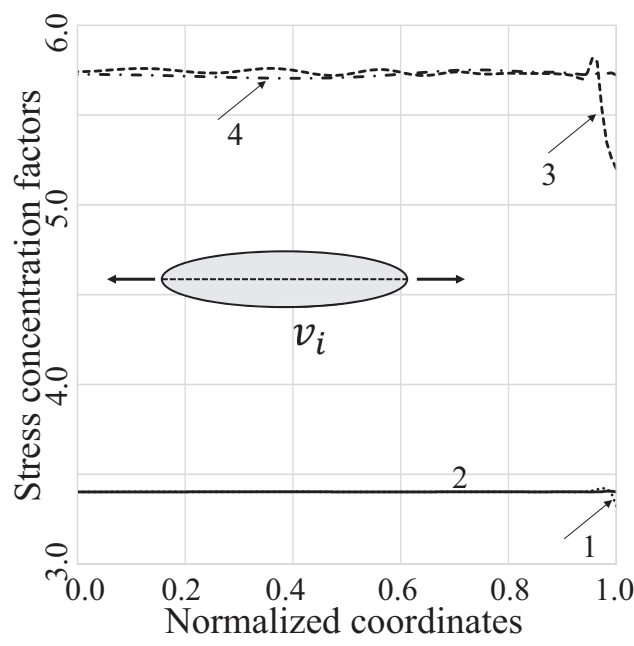

Fig. 3: Stress concentration factor $B_{i \mid 1111}\left(x_{1}\right)$ vs a normalized coordinate $x_{1} / R_{1}$.

With the non-essential restriction on space dimensionality $d$ and on the shape of inhomogeneities we will consider $2-D$ plane strain problems for composites reinforced by aligned infinite fibers with noncircular cross-section schematically presented in the Fig. 4 and described by the curve

$$
\begin{cases}(x-R+r)^{2}+(y-R+r)^{2}=r^{2}, & \text { for }\{|x|>R-r\} \cap\{|y|>R-r\}, \\ |x+y|+|x-y|=2 R, & \text { for }\{|x|<R-r\} \cup\{|y|<R-r\},\end{cases}
$$

which reduces to a circle and a square in the limiting cases $R=r=a$ and $r=0$, respectively. Normalized effective Young moduli $E_{11}^{*} / E^{(0)}$ are presented in Fig. 5 as the functions of the volume concentration of inclusions at $r=0.2,0.4,0.6,1 R$ (curves 1-4) estimated by the MEF. As can be seen, the estimations carried out for the different shape of inclusions defining by $r s$ are 395 essentially different at $c>0.6 ; E_{11}^{*}(0.8) / E^{(0)}$ at $c=0.8$ varies from 23.67 to 7.37 as $r$ varies from 0.2 to 1.0. In so doing, the corresponding values $E_{11}^{*}(0.8) / E^{(0)}$ 
estimated by the MT method at $c=0.8$ varies from 8.46 (curve 5) to 7.37 (curve 4) as $r$ varies from 0.2 to 1.0. The are known publications (see for references [6]) demonstrating equivalence of the MTM and MEF for composites with identical aligned homogeneous ellipsoidal heterogeneities $v_{i}$ with the homothetic excluded volume $v_{i}^{0}$. Because of this, the values $E_{11}^{*}(c) / E^{(0)}$ estimated by both the MTM and MEF for the circle inclusions $r=1$ are expectedly coincide with each other (curve 4 in Fig. 5). However, the estimations of $E_{11}^{*}(c) / E^{(0)}$ (curve 5) carried out by the MTM for $r=0.2$ are essentially different from the corresponding values evaluated by the MEF (curve 1). As can be seen, the MEF is basically sensitive with respect to the inclusion shape (defining by $\alpha$ ) than the MTM.

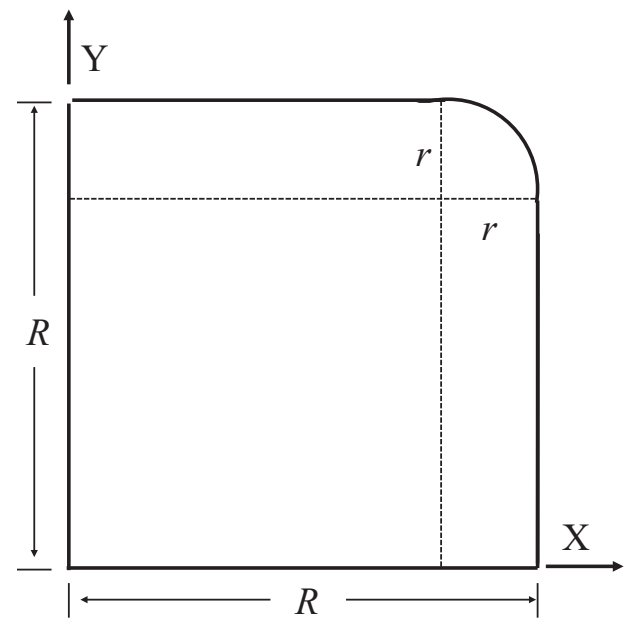

Fig. 4: Schematic of a noncircular inclusion characterized by the shape parameter $r / R$

\section{Conclusion}

In the present paper, numerical results were obtained for composites with statistically homogeneous field of homogeneous aligned prolate identical heterogeneities of non canonical shape. At first, the solution for one inclusion inside infinite medium was obtained by the MFS adapted for analysis of infinite domains. In the MFS one considers representations of the solution as a linear combination of point source fundamental tensors centered at some points 415 placed outside the boundary of interest. The vectorial coefficients of these linear combinations defining a coefficient matrix can be computed by imposing, on some boundary points (collocation points) at the interface, the boundary conditions of the transmission problem. Applications of the proposed techniques were illustrated through several numerical examples. Results obtained using the Tikhonov regularization techniques were compared to those obtained by the direct inversion of corresponding coefficient matrix for the different choices of 
the source sets and to analytical values. Several numerical examples for smooth 2D domains and smooth boundary data were presented in order to show the accuracy and feasibility of the method. Possible extensions to the 3D problem require only a placement of both the collocation and source points at the interface $\Gamma_{i}$ and the pseudo-boundaries $\hat{\Gamma}_{i}^{(0)}, \hat{\Gamma}_{i}^{(1)}$, respectively, are straightforward. The numerical evaluation of the effective Young modulus are shown for a statistically homogeneous composites reinforced by aligned identical homogeneous heterogeneities of noncanonical shape.

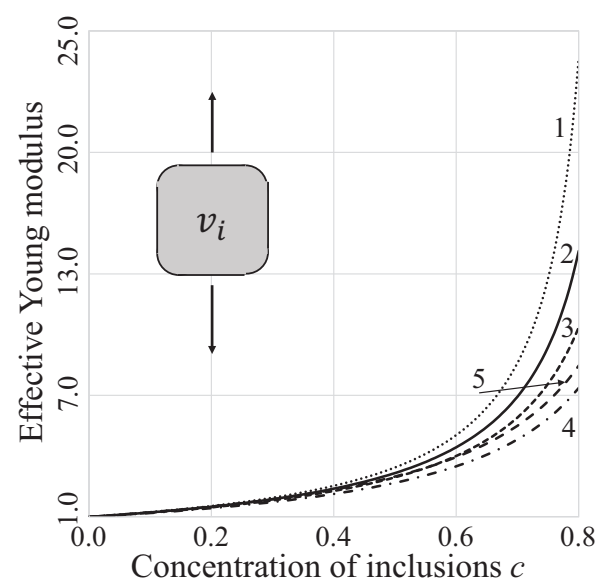

Fig. 5: Variation of the relative Young modulus $E_{11}^{*} / E^{(0)}$ as a function of a concentration of the

\section{Acknowledgments}

The author acknowledges the support of the US Office of Naval Research (Dr. William M. Mullins, contract: N00014-14-P-1255).

Appendix. The representations of the fundamental solutions (20) and $435 \quad(21)$

The tensors of the fundamental displacements, tractions, stresses, and strains (20), (21) have the following components $(d=2,3)$

$$
\begin{aligned}
G_{i j}(r) & =\operatorname{Cr}\left[(3-4 \bar{\nu}) \gamma(|\mathbf{x}|) \delta_{i j}+n_{i} n_{j}\right] \\
T_{i j}(\mathbf{x}, \mathbf{s}) & =-2 \mu C\left[\left((1-2 \bar{\nu}) \delta_{i j}+d n_{i} n_{j}\right) n_{k} n_{k}^{\Gamma}(\mathbf{x})\right. \\
& \left.+(1-2 \bar{\nu})\left(n_{i} n_{j}^{\Gamma}(\mathbf{x})-n_{j} n_{i}^{\Gamma}(\mathbf{x})\right)\right] \\
D_{i j k}(r) & =-2 \mu C\left[(1-2 \bar{\nu})\left(\delta_{i k} n_{j}+\delta_{j k} n_{i}-\delta_{i j} n_{k}\right)+d n_{i} n_{j} n_{k}\right], \\
\mathcal{E}_{i j k}(r) & =-C\left[(3-4 \bar{\nu}) \delta_{i j} n_{k}-\delta_{i k} n_{j}-\delta_{j k} n_{i}+d n_{i} n_{j} n_{k}\right],
\end{aligned}
$$


respectively, where

$$
\gamma(r)=\left\{\begin{array}{ll}
-\ln |\mathbf{x}| & \text { for } d=2, \\
1 & \text { for } d=3,
\end{array} \quad \bar{\nu}= \begin{cases}\nu, & \text { for 3-D and plane strain } \\
\frac{\nu}{1+\nu}, & \text { for plane stress problems }\end{cases}\right.
$$

Here $C=\left[4 \omega_{d} \mu(1-\bar{\nu}) r^{d-1}\right]^{-1}, \omega_{d}$ is the surface area of the unit sphere in $R^{d}$, and the tensor $\mathbf{T}(\mathbf{x}, \mathbf{s})$ is defined at the surface $\mathbf{s} \in S$ with the unit outward

normal $\mathbf{n}^{\Gamma}=\left(n_{1}^{\Gamma}, \ldots, n_{d}^{\Gamma}\right)^{\top}$ at $\mathbf{s} \in S, r \equiv|\mathbf{x}-\mathbf{s}|, r_{, i}=r_{i} / r=n_{i}$.

\section{References}

[1] Willis, J. R. Variational and related methods for the overall properties of composites. Advances in Applied Mechanics 1981; 21: 1-78.

[2] Mura, T. 1987. Micromechanics of Defects in Solids. Martinus Nijhoff, Dordrecht: 1987.

[3] Nemat-Nasser, S., Hori, M. Micromechanics: Overall Properties of Heterogeneous Materials. Elsevier, North-Holland: 1993.

[4] Torquato, S. Random Heterogeneous Materials: Microstructure and Macroscopic Properties. Springer-Verlag, NY, Berlin: 2002.

450 [5] Milton, G.W. The Theory of Composites. Applied and Computational Mathematics, vol. 6. Cambridge University Press: 2002.

[6] Buryachenko V. A. Micromechanics of Heterogeneous Materials. Springer, NY: 2007.

[7] Sejnoha, M., Zeman, J. Micromechanics in Practice. WIT Press, Southampton, UK: 2013.

[8] Dvorak, G.J. Micromechanics of Composite Materials. Springer, Dordrecht: 2013.

[9] Kröner, E. Berechnung der elastischen Konstanten des Vielkristalls aus den Konstanstanten des Einkristalls. Z. Physik. 1958; 151: 504-518.

${ }_{460}$ [10] Hill, R. 1965; A self-consistent mechanics of composite materials. J. Mech. Phys. Solids 1965; 13: 212-222.

[11] Mori, T., Tanaka, K. Average stress in matrix and average elastic energy of materials with misfitting inclusions. Acta Metall. 1973; 21: 571-574.

[12] Benveniste, Y. A new approach to application of Mori-Tanaka's theory in composite materials. Mech. Mater. 1987; 6: 147-157.

[13] Fan, X.-J., Phan-Thien, N., Zheng, R. Completed double layer boundary element method for periodic suspensions Z. angew. Math. Phys. 1998; 49: $167-193$.

[14] Liron, N., Barta, E. Motion of a rigid particle in Stokes flow: a new secondkind boundary-integral equation formulation J . Fluid Mech. 1992; 238: 579598.

[15] Fasshauer, G.E. Meshfree methods, Handbook of Theoretical and Computational Nanotechnology, M. Rieth and W. Schommers (eds.), American Scientific Publishers, 2006; 2 33-97. 
[16] Belytschko T, Krongauz Y, Organ D, Fleming M, Krysl P Meshless method: An overview and recent developments. Comput Methods Applied Mech Eng 1996; 139: 3-47.

[17] Goldberg, M.A., Chen, C.S. The method of fundamental solutions for potential, Helmholtz and diffusion problems. In M. A. Goldberg, editor, Boundary Integral Methods: Numerical and Mathematical Aspects Pineridge Press, Southampton, Boston, 1999; 103-176.

[18] Atluri, S.N. The meshless method (MLPG) for domain \& BIE discretizations Tech Science Press; 2004.

[19] Chen, W., Tanaka, M. A meshless, integration-free, and boundary-only RBF technique Computers \& Mathematics with Applications, 2002;43: 379-391.

[20] Fairweather, G., Karageorghis, A. The method of fundamental solutions for elliptic boundary value problems. Advances in Computational Mathematics 1998; 9: 69-95.

[21] Chen, K.H., Chen, J.T., Kao, J.H. Regularized meshless method for antiplane shear problems with multiple inclusions. Int. J. Numer. Meth. Engng 2008; 73: 1251-1273.

[22] Tikhonov, A.N., Arsenin, V. Y. Methods for Solving Ill-Posed Problems, Nauka, Moscow: 1986.

[23] Lin,J., Chen, W., Wang, F. A new investigation into regularization techniques for the method of fundamental solutions. Mathematics and Computers in Simulation 2011; 81: 1144-1152.

[24] Willis, J. R. Bounds and self-consistent estimates for the overall properties of anisotropic composites. J. Mech. Phys. Solids 1977; 25: 185-203.

[25] Buryachenko V. A. On the thermo-elastostatics of heterogeneous materials. I. General integral equation. Acta Mechanica. 2010; 213: 359-374.

[26] Buryachenko, V.A., On the thermo-elastostatics of heterogeneous materials. II. Analyze and generalization of some basic hypotheses and propositions. Acta Mech. 2010; 213: 375-398.

[27] Lekhnitskii, A.G. Theory of Elasticity of an Anisotropic Elastic Body. Holder Day, San Francisco; 1963.

[28] Hashin, Z., Shtrikman, S. On some variational principles in anisotropic and nonhomogeneous elasticity. J Mech Phys Solids 1962; 10: 335-342.

[29] Hill, R. Elastic properties of reinforced solids: some theoretical principles. $J$ Mech Phys Solids, 1963; 11: 357-372.

[30] Buryachenko V., Jackson T., Amadio G. Modeling of random bimodal structures of composites (application to solid propellant): I. Simulation of random packs. Comput. Model. Engng \& Sciences (CMES) 2012; 85: 379-416.

[31] Russel, W. B., Acrivos, A. On the effective moduli of composite materials: slender rigid inclusions at dilute concentrations. J Appl Math Phys (ZAMP) 1972; 23: 434-464.

[32] Chen, H.S., Acrivos, A. The effective elastic moduli of composite materials containing spherical inclusions at non-dilute concentrations. Int J Solids Struct 1978; 14: 349-364.

[33] Smyrlis, Y.-S. Mathematical foundation of the MFS for certain elliptic sys520 tems in linear elasticity. Numer. Math. 2009; 112: 319-340. 
[34] Balas, J., Sladek, J., Sladek, V. Stress Analysis by Boundary Element Methods. Elsevier, Amsterdam; 1989.

[35] Alves, C. J.S., Silvestre, A. L. Density results using Stokeslets and a method of fundamental solutions for the Stokes equations. Engineering Analysis with Boundary Elements 2004;28: 1245-1252.

[36] Martins, N.F.M., Rebelo, M. A meshfree method for elasticity problems with interfaces Applied Mathematics and Computation 2013; 219: 10732-10745.

[37] Young, D. L, Jane, S. J., Fan, C . M., Murugesan, K., Tsai, C. C. The method of fundamental solutions for 2D and 3D Stokes flows. J. Comput. $530 \quad$ Physics, 2006; 211: 1-8.

[38] Alves, C.J.S., Antunes, P.R.S. The method of fundamental solutions applied to the calculation of eigenfrequencies and eigenmodes of $2 \mathrm{D}$ simply connected shapes. Comput Mater Continua 2005;2: 251-66.

[39] Alves, C.J.S. On the choice of source points in the method of fundamental solutions. Engineering Analysis with Boundary Elements 2009;33: 1348-1361.

[40] Karageorghis, A., Smyrlis, Y.-S. Matrix decomposition MFS algorithms for elasticity and thermo-elasticity problems in axisymmetric domains. $J$. Computat. Applied Mathematics 2007; 206: 774-795.

[41] Hansen, P. C., Rank-Deficient and Discrete Ill-Posed Problems Numemcal Aspects of Linear Inversion, SIAM, Philadelphia, PA: 1998.

[42] Marin, L. A meshless method for solving the Cauchy problem in threedimensional elastostatics. Computers and Mathematics with Applications 2005; 50: $73-92$.

[43] Eshelby JD. The determination of the elastic field of an ellipsoidal inclusion, and related problems. Proc Roy Soc Lond 1957; A241: 376-396.

[44] Parnell, W.J. The Hill and Eshelby tensors for ellipsoidal inhomogeneities in the Newtonian potential problem and linear elastostatics. ArXiv preprint arXiv:1507.07165v1, 2015; 1-56.

[45] Dvorak, G. J., Benveniste, Y. (1992) On transformation strains and uniform fields in multiphase elastic media. Proc Roy Soc Lond, 1992; A437; 291-310.

[46] Lax, M. Multiple scattering of waves II. The effective fields dense systems. Phys. Rev. 1952; 85: 621-629. 